\title{
Annealing induced inversion of quantum dot fine-structure splitting
}

Cite as: Appl. Phys. Lett. 90, 181927 (2007); https://doi.org/10.1063/1.2737131

Submitted: 12 February 2007 . Accepted: 14 April 2007. Published Online: 04 May 2007

E. Margapoti, L. Worschech, A. Forchel, A. Tribu, T. Aichele, R. André, and K. Kheng

\section{ARTICLES YOU MAY BE INTERESTED IN}

Control of fine-structure splitting of individual InAs quantum dots by rapid thermal annealing Applied Physics Letters 90, 011907 (2007); https://doi.org/10.1063/1.2430489

Biexciton initialization by two-photon excitation in site-controlled quantum dots: The complexity of the antibinding state case

Applied Physics Letters 117, 134001 (2020); https://doi.org/10.1063/5.0011383

Challenge us.

What are your needs for periodic signal detection?
Watch

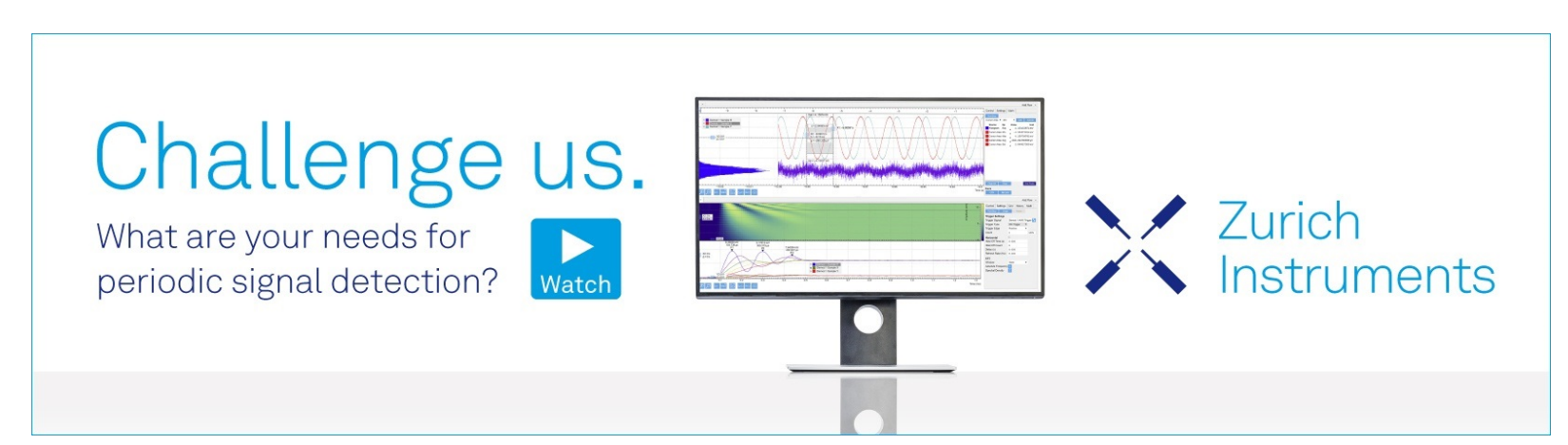

quantum dots: The

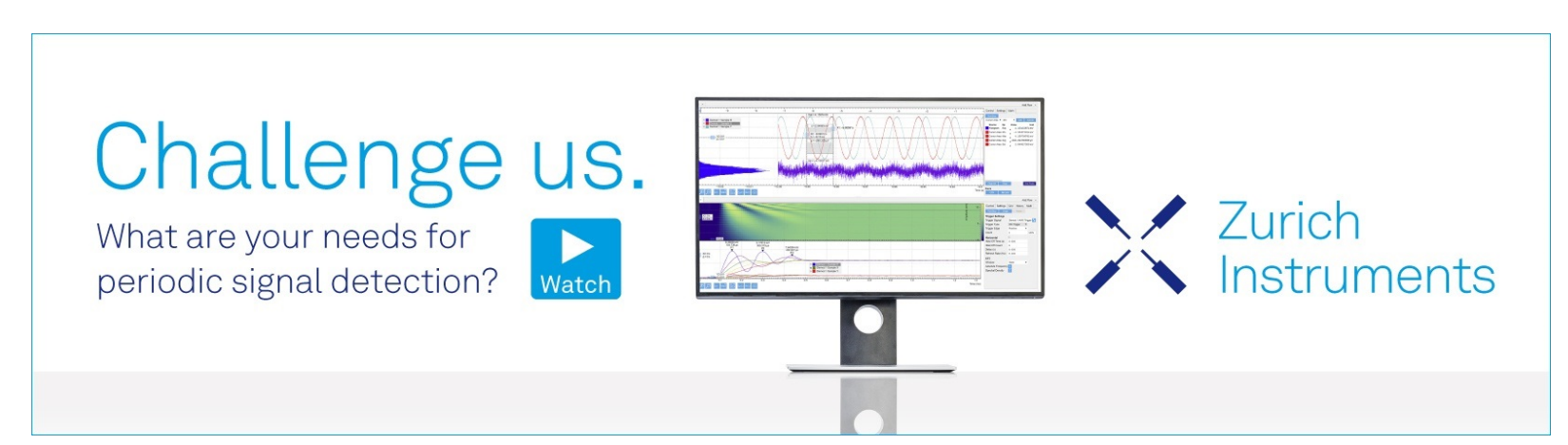

\section{舟}




\title{
Annealing induced inversion of quantum dot fine-structure splitting
}

\author{
E. Margapoti, ${ }^{\text {a) }}$ L. Worschech, and A. Forchel \\ Technische Physik, Physikalisches Institut, Universität Würzburg, Am Hubland, 97074 Würzburg, Germany
}

\begin{abstract}
A. Tribu, T. Aichele, R. André, and K. Kheng
CEA-CNRS "Nanophysique et Semiconducteurs," University J. Fourier-Grenoble, CEA-Grenoble, 17 Rue des Martyrs, 38054 Grenoble, France
\end{abstract}

(Received 12 February 2007; accepted 14 April 2007; published online 4 May 2007)

\begin{abstract}
By mapping the anisotropy fine-structure splitting of the exciton ground state in the luminescence spectra of individual $\mathrm{Cd}_{x} \mathrm{Zn}_{1-x}$ Se quantum dots, treated by postgrowth rapid thermal annealing (TA), a preferred in-plane axis of $\mathrm{Zn}-\mathrm{Cd}$ interdiffusion has been identified. In particular, a TA-induced sign reversal of the fine-structure splitting is demonstrated. Additionally, in the annealed quantum dots, the binding energy of the charged exciton reaches a maximum value when the fine-structure splitting is minimum. The studies demonstrate that by postgrowth thermal annealing the symmetry of individual quantum dot can be modulated. (c) 2007 American Institute of Physics.
\end{abstract}

[DOI: $10.1063 / 1.2737131]$

Quantum dots (QDs), often described as artificial atoms due to their discrete density of states, hold the potential to serve as the basic elements for quantum information processing. ${ }^{1,2}$ Semiconductor QDs are formed, among other methods, by self-assembly during epitaxial growth of latticemismatched crystal heterosystems. Often, kinetic limitations of epitaxial growth result in QD inhomogeneities and unintended asymmetries. Such asymmetries, e.g., an elongation of the QD along a particular crystallographic direction, ${ }^{3}$ cause a fine-structure splitting (FSS) of the radiative excitonic doublet. $^{4-6}$ The FSS has its origin in the electron-hole exchange interaction ${ }^{7,8}$ and manifests itself in a linear polarization of the photoluminescence (PL) emission along the direction of QD elongation, which in case of $\mathrm{Cd}_{x} \mathrm{Zn}_{1-x} \mathrm{Se}$ QDs is the [ $\left[\begin{array}{lll}1 & 1 & 0\end{array}\right]$ axis. Due to the FSS, the two possible cascades [gray and black arrows in Fig. 1(a)] for a biexciton $X^{2}$ to recombine into an exciton $X$ become energetically distinguishable, ${ }^{9}$ which hampers the generation of entangled photon pairs. ${ }^{6}$ Fine tuning of FSS can allow us to achieve indistinguishable photons from distinct QDs.

Postgrowth thermal annealing has been investigated for different semiconductor materials and demonstrated to improve their spectral quality and enhance their quantum efficiency significantly. ${ }^{10-13}$ For II-VI heterostructures, thermally activated interdiffusion has been observed in $\mathrm{CdTe} / \mathrm{CdMnTe}$ quantum wells and beneficially used to fabricate $\mathrm{CdTe} / \mathrm{CdMgTe}$ quantum dots under predefined $\mathrm{SiO}_{2}$ mask. ${ }^{14}$ However, reports on thermal annealing studies of II-selenide nanostructures are rare.

Recently, a drastically enhanced diffusion in thermally annealed $\mathrm{Cd}_{x} \mathrm{Zn}_{1-x} \mathrm{Se}$ QDs in small mesas, compared to unpatterned heterostructures, has been demonstrated and attributed to the presence of defects on the sidewalls of the small mesas. ${ }^{15,16}$ Activation energy of interdiffusion, as small as $1 \mathrm{eV}$, was determined from TA-induced spectral shifts of single-QD luminescence emission lines. Here, we demonstrate that modification of the QD form and composition, attributed to enhanced diffusion close to a mesa sidewall,

${ }^{a)}$ Electronic mail: emanuela.margapoti@physik.uni-wuerzburg.de also influences the shape asymmetry of the QDs, resulting in a modification of the FSS splitting.

The scenario is sketched in Fig. 1(c). Prior to thermal annealing (PTA), the QD is elongated along $\mathbf{H}$. Provided, TA results in a preferential interdiffusion along the perpendicular direction $\mathbf{V}$, the QD is expected to first become symmetric in shape, and subsequently elongate along $\mathbf{V}$ [after thermal annealing (ATA)]. Consequently, the FSS should exhibit a diminution, followed by a sign reversal. The binding energy of the charged exciton should also alter with a change of the QD symmetry, since the Coulomb interaction of the electrons is known to sensitively depend on the dimensions of lowdimensional systems. ${ }^{17-21}$ In this letter, we report the observation of both the aforesaid effects due to postgrowth ther-
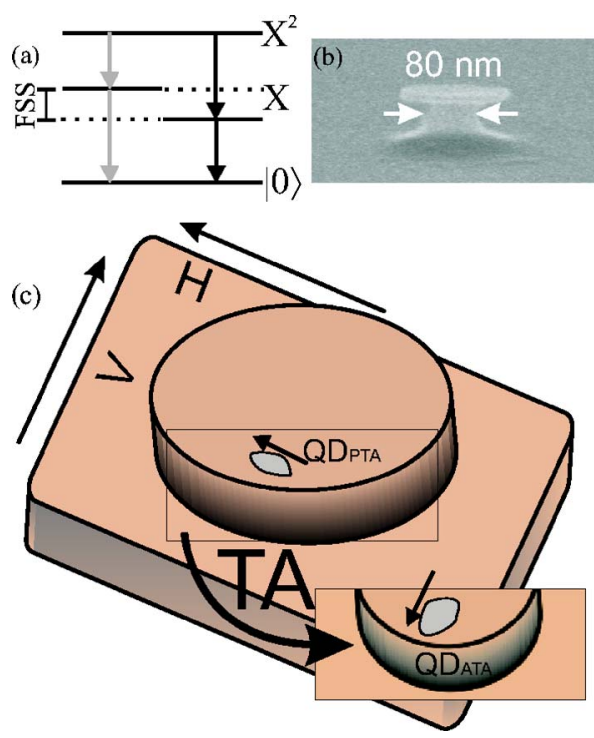

FIG. 1. (Color online) (a) Energy scheme of a QD exciton and biexciton with a FSS, caused by a QD asymmetry and an electron-hole exchange interaction. Due to a FSS, the recombination of the biexciton $X^{2}$ into the two linearly polarized exciton states $X$ can be energetically distinguished (gray and black arrows). (b) The electron microscope image of an etched mesa, taken prior the top mask removal. (c) A sketch of a QD elongated along the crystallographyc direction $\mathbf{H}$, close to the etched sidewall of the mesa, prior to TA (PTA). Defect-mediated interdiffusion, enhanced by TA, leads to a change of the QD elongation, after TA (ATA), along the $\mathbf{V}$ direction. 
mal annealing of $\mathrm{Cd}_{x} \mathrm{Zn}_{1-x} \mathrm{Se}$ QDs, embedded in small mesa structures.

The samples were prepared in the following way. First, a $1 \mu \mathrm{m} \mathrm{GaAs}$ buffer layer is grown by molecular beam epitaxy (MBE) on a $\left(\begin{array}{lll}0 & 0 & 1\end{array}\right) \mathrm{Zn}$ doped GaAs substrate, in order to obtain a proper surface for the subsequent growth. The sample is then transferred under vacuum to a II-VI MBE chamber. A $60 \mathrm{~nm} \mathrm{ZnSe} \mathrm{layer} \mathrm{is} \mathrm{grown} \mathrm{at} 280{ }^{\circ} \mathrm{C}$ with a growth rate of typically $0.4 \mathrm{ML} / \mathrm{s}$ ( $\mathrm{Zn}$ flux: 2.5 $\times 10^{-7}$ mbar, Se flux: $5 \times 10^{-7}$ mbar), controlled by reflection high-energy electron diffraction. Next, using atomic layer epitaxy, 3 ML CdSe was deposited, which is just below the critical thickness for strain relaxation. The sample was then slowly cooled down (within $2 \mathrm{~h}$ ) to $-10{ }^{\circ} \mathrm{C}$ and exposed to Se for $30 \mathrm{~min}$, creating a $50-100 \mathrm{~nm}$ thick layer of amorphous Se. Next, the sample temperature was slowly increased to $280{ }^{\circ} \mathrm{C}$ to desorb the amorphous Se. It is during this process, where the transition to quantum dot islands occurs. ${ }^{22}$ Finally the islands were overgrown with a $30 \mathrm{~nm}$ $\mathrm{ZnSe}$ capping layer.

In order to select single quantum dots, small mesa structures were fabricated by electron beam lithography and wet chemical etching, as shown in Fig. 1(b). Subsequently, the sample was thermally annealed on a hot plate for $30 \mathrm{~s}$ and at different annealing temperatures, $T_{A}=100,120,140,160$, and $180^{\circ} \mathrm{C}$. After each TA step, the low temperature PL spectra corresponding to the mesa were recorded, by immersing the sample in a $\mathrm{He}$ cold finger cryostat, reaching a temperature of $5 \mathrm{~K}$. The QDs were excited with the $405 \mathrm{~nm}$ line of a solid state continuous-wave laser. In order to investigate the linearly polarized QD emission, the PL was recorded with a linear polarizer placed in front of the monochromator, parallel to either $\mathbf{H}$ or $\mathbf{V}$, as indicated in Fig. 1(c). We identify the $\mathbf{H}$ and the $\mathbf{V}$ with the crystallographic directions $\left[\begin{array}{lll}1 & -1 & 0\end{array}\right]$ and $\left[\begin{array}{lll}1 & 1 & 0\end{array}\right]$, respectively.

In Fig. 2(a), PL spectra of a small mesa, recorded after two different annealing steps, are shown. As our discussion focuses on the changes of the FSS due to TA, the energy of the exciton $X_{1}$ was taken as the center of reference, with $E$ $=0$. In the spectra, an exciton line $X_{1}$ and the lines $X_{1}^{-}$and $X_{1}^{2}$, associated with the charged exciton and the biexciton, respectively, for $T_{A}=120$ and $180{ }^{\circ} \mathrm{C}$, are shown. At the highenergy side of the spectrum, the biexciton line of another QD can be seen $\left(X_{2}^{2}\right)$. For $T_{A}=120^{\circ} \mathrm{C}$, the lines associated with the $Q D_{1}$ are unpolarized and almost no FSS can be resolved. In contrast, at $T_{A}=180{ }^{\circ} \mathrm{C}$, the $X_{1}$ line shows a pronounced FSS, and the high and the low energy components are linearly polarized along $\mathbf{V}$ and $\mathbf{H}$, respectively. The reverse behavior is observed for the biexciton line, as shown schematically in Fig. 1(a).

Due to the singlet nature of the charged exciton, no preferential polarization can be observed for the $X_{1}^{-}$line. Clearly, TA leads to an increase of the FSS. Additionally, the binding energies of the charged exciton line change also significantly. In Fig. 2(b), an enlarged view of the exciton line, for $T_{A}=100{ }^{\circ} \mathrm{C}$ and $T_{A}=180{ }^{\circ} \mathrm{C}$, is presented. A comparison of these spectra shows that TA of small mesas leads also to a reversal of FSS. While the splitting of the exciton line is positive for $T_{A}=100{ }^{\circ} \mathrm{C}$, a negative $\mathrm{FSS}$ is seen for $T_{A}=180{ }^{\circ} \mathrm{C}$ [Fig. 3(b)].

We attribute this evolution of the FSS to a change of the QD shape due to mesa-sidewall-enhanced diffusion. Vacancies generated within mesa sidewalls enhance $\mathrm{Cd}-\mathrm{Zn}$ inter-
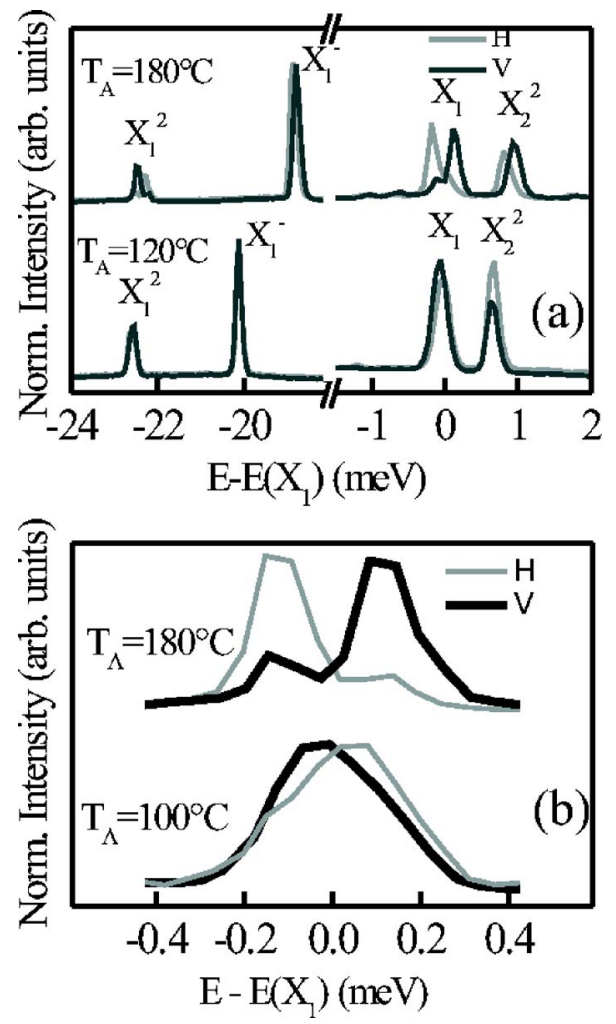

FIG. 2. (Color online) (a) PL spectra recorded from a $150 \mathrm{~nm}$ mesa for $T_{A}=180^{\circ} \mathrm{C}$ and $T_{A}=120^{\circ} \mathrm{C}$, where the single lines are associated with the exciton $X_{1}$, the biexcitons $X_{1}^{2}$ and $X_{2}^{2}$, and the charged exciton $X_{1}^{-}$. The prefixes 1 and 2 are used to identify the two different QDs. (b) A zoom of the sign reversal of FSS for the $\mathrm{QD}_{1}$ is shown for the two annealing temperatures.

diffusion in QDs in the vicinity of the mesa edge. Such vacancies are formed during the etching process. Vacancymediated enhanced interdiffusion ${ }^{15}$ has been observed previously in In-GaAs/InP quantum wells. ${ }^{23,24}$ In our case the density of the vacancies is larger close to the sidewalls, and consequently the QDs in close proximity of the edges would exhibit the FSS inversion. Thus, if a quantum dot

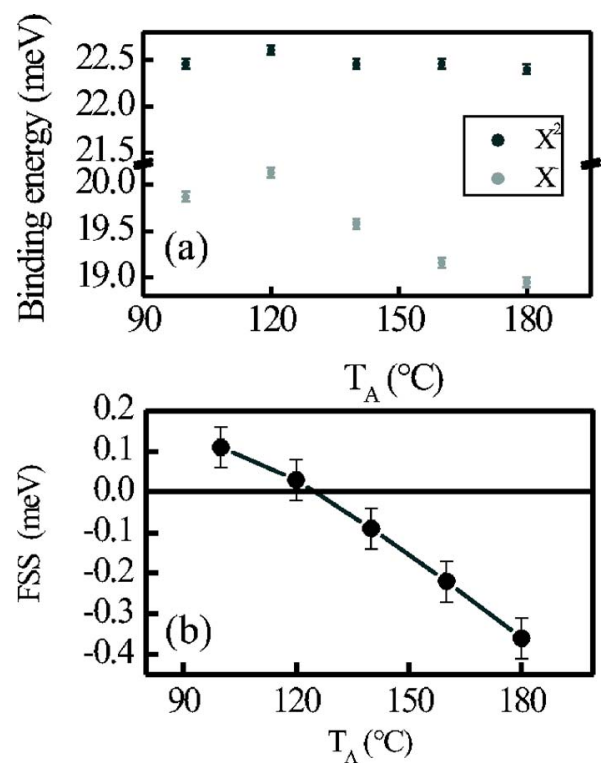

FIG. 3. (Color online) (a) Binding energies of the charged exciton and the biexciton (b) and fine-structure splitting versus annealing temperature. The error is $0.05 \mathrm{meV}$ and limited by the resolution of the setup. 
elongated collinear to $\mathbf{H}$, prior to thermal annealing, is located close to a sidewall with a surface vector collinear to $\mathbf{V}$, it undergoes an enhanced interdiffusion along $\mathbf{V}$ and therefore can change its shape.

In Fig. 3, the binding energies of the biexciton (black circles) and the charged exciton (gray circles) and the FSS (black circles) are shown versus different annealing temperatures $T_{A}$. From the spectra recorded at $T_{A}=120{ }^{\circ} \mathrm{C}$ [bottom curves in Fig. 2(a)], maximum binding energies of 22.5 and $19.8 \mathrm{meV}$ of the biexciton and charged exciton, respectively, were determined. Interestingly, the biexciton binding energy does not vary significantly due to the TA process. Only a rise of $0.14 \mathrm{meV}$, by raising $T_{A}$ from 100 to $120{ }^{\circ} \mathrm{C}$, and a subsequent drop of $0.06 \mathrm{meV}$, for $T_{A}=180^{\circ} \mathrm{C}$, were determined. In contrary to the case of the biexciton, the charged exciton is strongly influenced by TA. The binding energy of the charged exciton increases by $0.23 \mathrm{meV}$ for $T_{A}=120^{\circ} \mathrm{C}$, and thereafter decreases by $0.92 \mathrm{meV}$ for $T_{A}=180^{\circ} \mathrm{C}$.

We believe that this large variation of the binding energy, of the charged exciton, results due to an elongation of the quantum dot caused by TA. Normally, due to TA-induced increase in the QD size, we would expect a decrease of the binding energy. However, Fig. 3(a) shows an initial increase of the binding energy for $T_{A}=120{ }^{\circ} \mathrm{C}$, which coincides with the high symmetry point of the QD.

In conclusion we have demonstrated that the finestructure splitting of the radiative excitonic doublet of a single quantum dot can be modified by TA. With increasing temperature of TA, an initial diminution of the structure splitting, followed by its sign reversal and sharp increase in the opposite direction, has been recorded. We assign this observation to an enhanced interdiffusion close to the sidewalls of the small mesa due to defects generated during the etching process. Also, the binding energy of the charged exciton shows a maximum when the QD assumes the high symmetry configuration, and drastically reduces subsequently, when the QD undergoes an elongation. Such an experimental method, of tuning the FSS and controlling the QD anisotropy, is an important step toward the generation of entangled photons.

This work has been supported in part, by the financial funding from the Institut Universitaire de France and by the DFG through SFB 410 and by the state of Bavaria. One of the authors (T.A.) acknowledges financial funding from Deutscher Akademischer Austauschdienst DAAD.

${ }^{1}$ W. H. Jiang, D. A. Thompson, O. Hulko, B. J. Robinson, and P. Mascher, J. Vac. Sci. Technol. A 24, 700 (2006).

${ }^{2}$ A. Van der Ven and G. Ceder, Phys. Rev. Lett. 94, 045901 (2005).

${ }^{3}$ T. Aichele, I. C. Robin, C. Bougerol, R. Andre, S. Tatarenko, and G. Van Tendeloo, J. Cryst. Growth 301-302, 281 (2007).

${ }^{4}$ R. Seguin, A. Schliwa, S. Rodt, K. Pötschke, and D. Bimberg, Phys. Rev. Lett. 95, 257402 (2005).

${ }^{5}$ T. Flissikowski, A. Hundt, M. Lowisch, M. Rabe, and F. Henneberger, Phys. Rev. Lett. 86, 3172 (2001).

${ }^{6}$ R. M. Stevenson, R. J. Young, P. Atkinson, K. Cooper, D. A. Ritchie, and A. J. Shields, Nature (London) 439, 179 (2006)

${ }^{7}$ E. Blackwood, M. J. Snelling, S. R. Andrews, C. T. B. Foxon, and R. T. Harley, Phys. Rev. B 50, 14246 (1994).

${ }^{8}$ M. Chamarro, C. Gourdon, O. Lublinskaya, A. I. Ekimov, and P. Lavallard, Phys. Rev. B 53, 1336 (1996).

${ }^{9}$ V. D. Kulakovskii, G. Bacher, R. Weigand, T. Kümmell, E. Borovitskaya, K. Leonardi, D. Hommel, and A. Forchel, Phys. Rev. Lett. 82, 1780 (1999).

${ }^{10}$ P. E. Smith, S. H. Goss, S. T. Bradley, M. K. Hudait, Y. Lin, S. A. Ringel, and L. J. Brillson, J. Vac. Sci. Technol. B 22, 554 (2004).

${ }^{11}$ R. Leon, Y. Kim, C. Jagadish, M. Gal, J. Zou, and D. J. H. Cockayne, Appl. Phys. Lett. 69, 1888 (1996).

${ }^{12}$ A. Krost, J. Christen, N. Oleynik, A. Dadgar, S. Deiter, J. Bläsing, A. Krtschil, D. Forster, F. Bertram, and A. Diez, Appl. Phys. Lett. 85, 1496 (2004).

${ }^{13}$ D. O. Yi, I. D. Sharp, Q. Xu, C. Y. Liao, J. W. Ager III, J. W. Beeman, Z. Liliental-Weber, K. M. Yu, D. Zakharov, E. E. Haller, and D. C. Chrzan, Mater. Res. Soc. Symp. Proc. 821, 16 (2004).

${ }^{14}$ S. Zaitsev, M. K. Welsch, H. Schömig, G. Bacher, V. D. Kulakovskii, A. Forchel, B. König, C. R. Becker, W. Ossau, and L. W. Molenkamp, Semicond. Sci. Technol. 16, 631 (2001).

${ }^{15}$ Martin Eden Glicksman, DIFFUSION IN SOLIDS Field Theory, SolidState Principles (Wiley-Interscience, New York, 2000), p. 224.

${ }^{16}$ E. Margapoti, L. Worschech, T. Slobodskyy, L. W. Molenkamp, and A. Forchel, J. Appl. Phys. 100, 113111 (2006).

${ }^{17}$ G. V. Astakhov, V. P. Kochereshko, W. Ossau, W. Faschinger, J. Puls, F. Henneberger, S. A. Crooker, Q. McCulloch, D. Wolverson, N. A. Gippius, A. Waag, and D. R. Yakovlev, Phys. Rev. B 65, 165335 (2002).

${ }^{18}$ I. A. Akimov, K. V. Kavokin, A. Hundt, and F. Henneberger, Phys. Rev. B 71, 075326 (2005).

${ }^{19}$ T. Otterburg, D. Y. Oberli, M.-A. Dupertuis, N. Moret, E. Pelucchi, B. Dwir, K. Leifer, and E. Kapon, Phys. Rev. B 71, 033301 (2005).

${ }^{20}$ A. V. Filinov, C. Riva, F. M. Peeters, Yu. E. Lozovik, and M. Bonitz, Phys. Rev. B 70, 035323 (2004).

${ }^{21}$ M. Bayer, T. Gutbrod, V. D. Kulakovskii, A. Gorbunov, M. Michel, R. Steffen, K. H. Wang, and A. Forchel, Phys. Rev. B 58, 4740 (1998).

${ }^{22}$ I.-C. Robin, R. André, C. Bougerol, T. Aichele, and S. Tatarenko, Appl. Phys. Lett. 88, 233103 (2006).

${ }^{23}$ J. Oshinowo, D. Grützmacher, M. Stollenwerk, M. Heuken, K. Heime, and A. Forchel, Appl. Phys. Lett. 60, 2660 (1993).

${ }^{24}$ J. H. Marsh, Semicond. Sci. Technol. 8, 1136 (1993). 